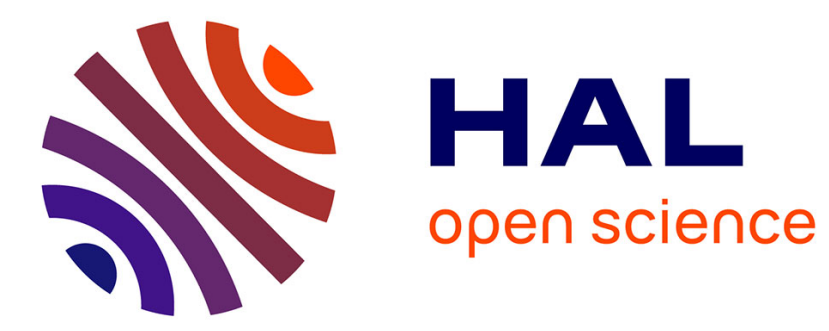

\title{
Dépassement et transformation de soi. Comment devenir pratiquant d'ultrafond ? \\ Sandrine Knobé
}

\section{To cite this version:}

Sandrine Knobé. Dépassement et transformation de soi. Comment devenir pratiquant d'ultrafond ?. Terrains et Travaux: Revue de Sciences Sociales, 2007, 12, pp.11-27. 10.3917/tt.012.0011. hal02536179

\section{HAL Id: hal-02536179 \\ https://hal.science/hal-02536179}

Submitted on 7 Apr 2020

HAL is a multi-disciplinary open access archive for the deposit and dissemination of scientific research documents, whether they are published or not. The documents may come from teaching and research institutions in France or abroad, or from public or private research centers.
L'archive ouverte pluridisciplinaire HAL, est destinée au dépôt et à la diffusion de documents scientifiques de niveau recherche, publiés ou non, émanant des établissements d'enseignement et de recherche français ou étrangers, des laboratoires publics ou privés. 


\section{Dépassement et transformation de soi. Comment devenir pratiquant d'ultrafond?}

\section{Introduction}

Dans les années 1980 se développent un certain nombre d'activités sportives qualifiées d'extrêmes (saut à l'élastique, triathlon, ski extrême...). Selon certains auteurs, cette recherche d'extrême constituerait une quête de limites (physiques) dans un environnement social caractérisé par une absence de repères sociaux et moraux (Baddeley, 2002). L'individu éprouverait son existence par la confrontation physique à ses limites. Ces pratiques apparaissent ainsi dans un contexte social où la " passion du risque" (Le Breton, 1991) et le «culte de la performance" (Ehrenberg, 1991) seraient les maîtres mots.

Loin d'être réservées à une élite sportive, elles encouragent tout un chacun à relever le défi et à essayer de dépasser ses limites (Barthélémy, 1998). Disputées en individuel ou par équipe, ces épreuves sont extrêmes par leur nature même (activité d'endurance sur des distances exceptionnellement longues) et par les conditions de leur déroulement (nature "hostile» - désert, jungle - autosuffisance alimentaire...). Le marathon des sables, épreuve de course à pied de plusieurs jours dont la particularité principale est de se dérouler en autosuffisance alimentaire ${ }^{1}$ en plein désert sud marocain, fait partie de ces courses extrêmes (Barthélémy, 2002). Par des températures avoisinant parfois les $50^{\circ} \mathrm{C}$, les concurrents parcourent chaque jour des distances oscillant entre 20 et 80 kilomètres (pour un total d'environ 240 kilomètres en 6 étapes). Mêlant tourisme sportif, performance et survie, le marathon des sables ${ }^{2}$ a connu une croissance exponentielle passant de 23 concurrents en 1986 à plus de 700 en 2005.

Un effet de mode ou de nouveauté permet sans doute, dans une certaine mesure, de comprendre l'engouement suscité par ces activités

\footnotetext{
1 Elle implique le portage d'un sac à dos pesant entre 7 et 10 kilos.

2 Organisé pour la première fois en 1986, le marathon des sables est une course par étapes se déroulant dans le désert.. Des tentes berbères pour huit personnes sont montées par l'organisation et l'eau est fournie aux ravitaillements. Les participants disposent d'un roadbook indiquant le tracé et le kilométrage (entre 20 et $80 \mathrm{~km}$ ) de chaque étape avec l'emplacement des postes de contrôle et de ravitaillement. Un temps maximum est prévu pour chaque étape sous peine de disqualification. Une équipe médicale participe à l'encadrement des coureurs (soins des blessures liées à la course - ampoules, irritations cutanées, déshydratation, coup de chaleur..). Le jour suivant la fin de la course est consacré à la remise des prix et à la visite de Ouarzazate.
} 
d'ultrafond $^{3}$, mais il n'explique pas ce que peut représenter une telle pratique pour un individu donné. En effet, il n'est pas anodin de s'élancer sur des courses qui franchissent allègrement le seuil des 42 kilomètres du traditionnel marathon (Segalen, 1994). Support d'enjeux variés (sportifs, symboliques, personnels...), les attentes et les ressorts de la motivation de ces coureurs semblent pluriels. Il ne s'agit pas uniquement de pratiquer une activité sportive, mais bien plus, de participer à une aventure humaine où la quête du dépassement de soi constitue un moteur important (Quéval, 2004).

Mais comment devient-on "sportif de l'extrême"? S'il n'y a pas de parcours type amenant à participer à un marathon des sables, il existe probablement des éléments structurants caractéristiques de ces engagements "hors du commun". S'engager sur un marathon des sables n'est pas une décision prise sur un "coup de tête", ne serait-ce que parce qu'elle suppose un investissement financier ${ }^{4}$ et temporel non négligeable. Son aspect réfléchi rend l'analyse doublement intéressante en excluant les engagements de pur hasard et en renforçant l'importance d'un tel engagement aux yeux mêmes de l'individu concerné. Quelles sont alors les conditions susceptibles d'inciter un individu à participer au marathon des sables ? S'y inscrire présupposet-il des dispositions particulières? Qu'il s'agisse d'une décision strictement personnelle ou d'une réponse à une forte sollicitation extérieure (d'amis coureurs par exemple), cet engagement soulève des interrogations d'ordre général, comme l'existence ou non de dispositions, c'est-à-dire de propriétés incorporées sous forme de schèmes de perception, de pensée et d'action (Bourdieu, 1980), à l'égard de ce type de pratique, et d'ordre particulier, comme les significations singulières qu'il prend pour un individu donné.

Pour appréhender les raisons d'un tel engagement, il convient de revenir sur le parcours qui y mène et dont les différentes étapes ne suivent pas nécessairement un chemin linéaire, en fonction de ce que représente une telle pratique dans l'histoire propre de chaque individu. Il importe surtout de saisir ce qui, dans un parcours biographique donné, est en mesure d'orienter l'individu vers une pratique comme l'ultrafond. Pour certains, cet engagement constitue une continuité dans un parcours sportif en course à pied où les distances s'allongent progressivement dans des défis souvent renouvelés. Des dispositions à

\footnotetext{
3 "L'ultrafond " désigne les courses dont les distances sont supérieures à celles du marathon (42,195 km et). Une revue, baptisée Ultrafondus, consacrée à ce type de courses, a vu le jour en mai 2003. (cf. www.ultrafondus.com).

4 Les frais d'engagement au Marathon des Sables s'élevaient à 2400 euros en 2004.
} 
l'ascèse et à l'effort physique prolongé encourageraient de tels parcours. Pour d'autres, il représente davantage une rupture avec des conditions sociales d'existence où le sport, et plus encore l'ultrafond, n'avaient que peu, voire pas du tout, de place. Quelle est alors l'origine d'une telle rupture?

L'objet de cet article est ainsi d'analyser deux points essentiels du parcours de ces coureurs : leur entrée dans l'ultrafond et les effets d'un tel engagement. Loin de chercher à donner un sens général à ces engagements extrêmes, nous essayerons, au contraire, de saisir les variations individuelles d'un tel engagement. L'extrême sera ici pris comme révélateur de continuité(s) et de rupture(s) biographiques traduisant la dialectique qui s'établit entre des dispositions individuelles et des contextes sociaux, c'est-à-dire des univers sociaux différenciées -la famille, l'école, l'univers professionnel, le monde du sport, etc. - où l'acteur vit des expériences variées (Lahire, 1998) ${ }^{5}$.

\section{Méthodologie}

Les résultats présentés ici s'appuient sur trois sources:

- les archives $^{6}$ du marathon des sables permettent de circonscrire les principales caractéristiques des participants essentiellement en termes de nationalité, d'âge et de sexe.

- un questionnaire ${ }^{7}$ a été administré aux participants français à l'édition $2004 \mathrm{du}$ marathon des sables. 123 des 186 concurrents français ont répondu à ce questionnaire cherchant à rendre compte de leurs propriétés sociales et sportives.

- des récits de pratiques ${ }^{8}$ (Bertaux, 1997) ont été recueillis avec des participants aux éditions 2003, 2004 et/ou 2005 du marathon des sables. Ils concernent quarante hommes et dix femmes. L'accent a été mis sur le récit de leur parcours (sportif) jusqu'au marathon des sables et sur les significations que peut revêtir un tel engagement. Une place importante a également été accordée à leur histoire de vie au sens plus large (trajectoires scolaire, professionnelle et familiale) afin de replacer une telle participation dans le parcours biographique de l'individu.

5 "Les répertoires de schèmes d'action (d'habitudes) sont des ensembles abrégés d'expériences sociales, qui ont été construitsincorporés au cours de la socialisation antérieure dans des cadres sociaux limités-délimités, et ce que chaque acteur acquiert progressivement et plus ou moins complètement, ce sont autant des habitudes que le sens de la pertinence contextuelle (relative) de leur mise en oeuvre " (Lahire, 1998, p.42)

6 Consultables sur le site Internet officiel de la course : www.darbaroud.com.

$7 \mathrm{Cf}$. annexe 1.

$8 \mathrm{cf}$. annexe 2. 


\section{Trajectoires vers l'ultrafond : logiques dispositionnelles et contextuelles}

Des dispositions à supporter une grande souffrance physique, à endurer des efforts longs dans des conditions de températures extrêmement élevées, à s'astreindre à une préparation rigoureuse trouvent au marathon des sables un lieu d'expression privilégié. Les parcours qui mènent à l'ultrafond ne sont pas tous identiques et dépendent de deux éléments principaux: l'existence de dispositions initiales à l'effort extrême acquises au cours de la socialisation primaire et de la socialisation secondaire (Berger \& Luckmann, 1989), surtout dans le monde sportif, et de conditions sociales rendant cet engagement plausible (Lahire, 2002). Ainsi, se dégagent trois profils de coureurs qui se distinguent en fonction de leur histoire sportive. L'analyse des modes d'entrée dans l'ultrafond révèle ensuite les influences exercées par les contextes de socialisation à l'origine d'expériences variées dans différents univers sociaux, en particulier le milieu familial, mais aussi les groupes de pairs et notamment, pour les pratiquants d'ultrafond, le milieu sportif (Lahire, 1998).

\section{Socialisation sportive "forte " et socialisation sportive "faible"}

Certains participants au marathon des sables, que nous qualifierons d'initiés, présentent un parcours à socialisation sportive "forte». Ils débutent la course à pied relativement jeunes, souvent pendant l'adolescence où ils ont fait partie d'un club d'athlétisme et alternaient courses sur piste et sur route (du $10 \mathrm{~km}$ au marathon). Avec l'ancienneté acquise dans la discipline, ils cherchent à varier les courses et à se lancer de nouveaux défis. Les participants au marathon des sables sont majoritairement des coureurs expérimentés puisqu'en moyenne ils courent depuis quatorze ans et que seul un peu plus de $6 \%$ des concurrents questionnés pratiquent la course à pied depuis moins de cinq ans. Au cours de leurs nombreuses années de pratique, ces coureurs ont acquis un savoir-faire et un savoir-être propices à l'effort de longue durée. Ils évoquent d'ailleurs souvent la nécessité de savoir gérer son effort. Cette gestion s'acquiert progressivement par les coureurs, dans le domaine de la course à pied, voire dans d'autres activités sportives pratiquées auparavant comme le cyclisme, le 
triathlon ou l'aviron. Plusieurs coureurs interrogés ont ainsi " reconverti ", dans la course à pied, des compétences sportives acquises dans d'autres disciplines, caractérisées par des efforts en endurance (Pociello, 1981).

Exemple de Laurent: "J'ai 34 ans et j'ai pratiqué l'aviron pendant 22 ans dont certaines années à haut niveau. La course à pied était un complément de cette activité nautique, il m'arrivait donc de courir une ou deux fois par semaine... Lorsque j'ai arrêté l'aviron, j'ai fait de l'athlétisme pendant 2 ans. Puis depuis 2 ans, j'ai monté une équipe de raid aventure lyonnaise team Planete-Tonique.com et je pratique donc les raids multisports extrêmes. On touche à tout, VTT, kayak, course à pied, orientation, cordes, roller... pendant des raids de 4 heures à 1 journée voire 2, 3, 4 jours... J'ai le MDS [marathon des sables] en rêve depuis des années et avec des amis on a monté un dossier pour être sponsorisés. Du coup ça a marché et on s'est retrouvé dans le désert tous frais payés. De ce fait, je me suis bien préparé et en avril 2004, je participai à mon premier MDS et j'en suis revenu avec une 35ème place".

Les trajectoires menant au marathon des sables ne sont pas toutes aussi continues et linéaires. Ainsi certains coureurs pratiquaient déjà une activité sportive (course à pied ou autre) durant leur jeunesse et adolescence. Puis, des contraintes personnelles ou professionnelles (Mignon \& Truchot, 2002) les ont amenés à abandonner toute pratique sportive, parfois de longues années durant. Des conditions et des attentes particulières, notamment un souci hygiéniste ${ }^{9}$ à l'approche de la quarantaine ${ }^{10}$ (Smith, 2000), les conduiront à (re-)commencer la course à pied. Que leur parcours sportif soit continu ou discontinu, ces coureurs initiés semblent avoir acquis des dispositions sportives s'exprimant dans leur rapport très compétitif 11 à la course à pied. Généralement membre d'un club d'athlétisme, ils cherchent constamment à améliorer leurs performances et leurs classements.

D'autres participants au marathon des sables, que nous qualifierons de novices, présentent un parcours a priori plus atypique dans la mesure où avant de commencer la course à pied, souvent tardivement (vers 3540 ans), ils n'avaient jamais pratiqué d'activité sportive. Ils opèrent

\footnotetext{
9 Pour $97 \%$ des coureurs interrogés, le "sport, c'est rester en forme, en bonne santé ".

10 La moyenne d'âge des concurrents français engagés en 2004 est de 42 ans.

11 Au-delà de 15 ans d'ancienneté en course à pied, ils sont $43 \%$ a estimé que "le sport, c'est gagner, faire de la compétition " contre seulement $17 \%$ en-dessous de 5 ans d'ancienneté.
} 
ainsi une véritable rupture sportive, passant d'un statut de non-sportif à une pratique régulière et progressivement intensifiée de la course à pied. Au départ, ils courent généralement seuls, sous la forme d'un footing hebdomadaire, sans intention compétitive. Leur "faible" socialisation sportive initiale renforce d'autant plus la rupture effectuée faisant pénétrer ces coureurs dans un "nouveau monde". Le passage $\mathrm{du}$ footing hebdomadaire à l'ultrafond se fait assez rapidement, parfois en l'espace de deux ou trois ans. L'entrée dans l'ultrafond semble à première vue prendre un caractère fortuit, car elle est inattendue pour un individu initialement non-sportif, et influencée par des contextes de socialisation spécifiques.

\section{Modes d'entrée dans l'ultrafond : l’importance du contexte}

Il s'avère de fait nécessaire de questionner les modes d'entrée dans l'ultrafond. Les coureurs d'ultrafond présentent en général un parcours en course à pied où les distances se sont allongées au fil du temps: d'abord un premier $10 \mathrm{~km}$, ensuite un semi-marathon, un marathon puis une première course d'ultrafond $\left(100 \mathrm{~km}\right.$, trails ${ }^{12}$, marathon des sables...). Peu de coureurs ${ }^{13}$ se lancent dans l'ultrafond sans n'avoir jamais participé à un marathon. Ces derniers, dont le passage à l'ultrafond est plus rapide, privilégient le côté " nature" des courses d'ultrafond et insistent sur leur distanciation vis-à-vis des préoccupations chronométriques, omniprésentes selon eux, au niveau des courses sur route.

Qu'il s'agisse de coureurs novices ou initiés, l'entrée dans l'ultrafond semble influencée par des conditions sociales particulières notamment des contextes de socialisation spécifiques. Une "forte» socialisation sportive, amenant certains coureurs a développé leurs dispositions aux courses de longue durée, peut constituer un contexte favorable à l'entrée dans l'ultrafond sous l'influence des partenaires de course et l'émulation qui naît des interactions entre coureurs. Ainsi, certains s'y inscrivent sous l'influence d'un ami coureur qui, parfois, y a déjà participé au moins une fois. D'autres fois, c'est un membre de l'entourage familial, amical $^{14}$ ou professionnel qui est à l'origine d'un tel engagement.

\footnotetext{
12 Il s'agit de courses sur terrain accidenté (petits chemins de campagne, forestiers ou montagnards). Ce type de courses, où le dénivelé peut être très élevé, se court sur des distances de quelques dizaines de kilomètres (de 20 à 50 km). Au-delà, on parle d'ultraTrails.

$1379 \%$ des participants au marathon de sables ont couru un ou plusieurs marathons.

14 Ce qui rejoint les résultats de l'enquête sur Les pratiques sportives en France (2002) où $34 \%$ des pratiquants affirment avoir été encouragé par leur conjoint ou concubin et $22 \%$ par un ami.
} 
Pour d'autres coureurs enfin, l'entrée dans l'ultrafond fera suite à un événement vécu de manière traumatique comme la perte d'un conjoint ou la survenue d'une maladie grave. La course à pied et plus particulièrement la participation à un marathon des sables revêtent alors des significations personnelles très fortes. À la souffrance endurée dans des situations non désirées, fait place la souffrance maîtrisée dans une quête de dépassement de soi synonyme de santé et de liberté. De tels événements déclencheurs amènent l'individu à relativiser les difficultés de certaines courses face à celles rencontrées hors du cadre sportif.

\section{ISABELLE - née en 1956 - 519 ème au marathon des sables 2004 Du cancer au désert}

Elle débute la course à pied en 2002 dans une optique de maintien en forme. Très rapidement elle constate ses progrès, s'aligne sur diverses courses et passe à l'ultrafond sous l'influence de ses amis coureurs au sein de son club d'athlétisme qui compte, chaque année, plusieurs participants au marathon des sables. C'est ainsi que, encouragée par ses amis et sa famille, elle s'y élance elle-même en 2004 avec pour seul objectif de finir. Certes, il s'agissait là de relever un défi corporel et sportif, mais bien plus de remporter une victoire en prouvant à soimême et aux autres sa capacité à réaliser des choses " extraordinaires " après avoir été confrontée à la mort.

En effet, l'engagement dans l'ultrafond fait suite à un cancer du sein déclaré en 1992. Après quatre opérations en un peu plus d'une année, elle est considérée comme guérie. Elle commence alors une psychothérapie, et change de profession trois ans après. En 2002, année même où elle débute la course à pied, elle redevient infirmière, métier qu'elle avait délaissé quelques années afin d'enseigner en école maternelle. Ces changements participent d'une reconstruction identitaire après une maladie létale qui marque le corps et le mental. Redevenir maître de son corps après en avoir perdu le contrôle pendant la maladie semble primordial.

$\mathrm{Au}$ moment de sa troisième opération, par exemple, ses sentiments sont mitigés et l'idée de mort toujours présente : "cette fois-ci, je m'étais dit, à quoi bon! J'étais prête à tout laisser tomber, même à mourir. Et puis les visites, les liens me font repartir dans la vie et je me relève après cette troisième intervention. Mais, quelques mois après, il faut réintervenir... J'accepte de me faire opérer à la seule condition que ce soit la dernière fois car je n'ai plus d'énergie. Cette fois-ci c'est la bonne, et je décide de 
tourner la page. Excepté un mois d'arrêt maladie, je continue à travailler. Travailler c'est vivre, et vivre... c'est ne pas mourir". La course à pied, comme le travail, sont pour elle le meilleur moyen de se sentir vivante. Le fait d'être sponsorisée par l'Institut Marie Curie ${ }^{15}$ afin de participer au marathon des sables constitue aussi tout un symbole dont la force du message réside dans la capacité à vaincre le désert après avoir vaincu le cancer.

\section{Effets pluriels d'un engagement extrême}

L'entrée dans l'ultrafond se différencie en fonction, d'une part, de dispositions individuelles à l'égard de ce type d'activité et, d'autre part, de conditions sociales spécifiques. C'est l'interaction entre ces deux éléments, tout au long des parcours biographiques individuels, qui permet de comprendre les variations de modes d'entrée dans l'ultrafond. La plus ou moins "forte" ou "faible» socialisation sportive des coureurs, à l'origine de dispositions différenciées à l'égard de la course à pied, de l'entraînement, de la compétition éclaire également pertinemment les effets différenciés d'un tel engagement.

\section{Des ascètes permanents : renforcement des dispositions à l'effort}

La pratique de l'ultrafond permet ainsi, pour certains coureurs, de renforcer des dispositions à l'ascétisme et à l'effort soutenu acquises, pour une large part, au cours de la socialisation primaire. En effet, l'impact d'une éducation où l'effort est valorisé n'est pas négligeable. Beaucoup de coureurs insistent sur l'importance que revêtaient, aux yeux de leurs parents, le fait de s'astreindre à des efforts, parfois longs et difficiles, afin d'atteindre des objectifs donnés. La réussite scolaire en est sans doute l'exemple paroxystique. La valorisation de l'ascèse pouvait, en outre, être renforcée par l'influence d'une éducation religieuse aux observances strictes. Le passage par un établissement scolaire privé à vocation religieuse n'est sans doute pas sans effet sur les conceptions de l'effort et de la souffrance.

15 Fondation privée à but non lucratif, implantée à Paris, dont les missions sont le diagnostic, le traitement et la recherche contre le cancer. 
Des dispositions à l'effort extrême

Il a commencé la course à pied en 1983, fera son premier marathon en 1987 et établira, quelques années plus tard, à $2 \mathrm{~h} 52$ son record sur cette même distance. Mais son envie est d'aller «toujours plus haut, toujours plus fort". Le marathon des sables constitue alors un défi de taille. La décision d'y participer, en 2003, arrive à un moment où Cyrille s'interroge sur lui-même et en particulier sur ses capacités professionnelles: "j'ai eu certaines difficultés professionnelles et il me paraissait important de me prouver que j'étais encore capable de faire quelque chose, même si c'était extra-professionnel ".

Étant jeune, il a pratiqué le judo de 8 à 12 ans et l'athlétisme dans le cadre scolaire. Ce n'est qu'à l'âge de 24 ans qu'il démarre intensément la course à pied. Il a été, un temps, entraîneur dans un club d'athlétisme. Maintenant, il planifie ses propres séances et celles d'une de ses filles, âgée de 16 ans. Ces cinq filles sont d'ailleurs très sportives, ce à quoi il prête une attention particulière: "chez nous, il y a un tiercé gagnant : école, musique et sport. Je ne sais pas vraiment s'il est gagnant mais c'est comme ça et pour tous les enfants... L'esprit de compétition souffle constamment dans la famille dans ces trois domaines... Nous estimons qu'il s'agit du meilleur moyen pour nos enfants de se préparer aux épreuves qu'ils auront à affronter arrivés à l'âge adulte... C'est comme ça depuis leur naissance et pour toute la famille. Ce qui est valable pour l'école (qui est obligatoire dans toutes les familles), est appliqué chez nous à la musique et au sport. C'est obligatoire et personne n'y réchappe et ce jusqu'au baccalauréat minimum ".

Issu d'un milieu aisé (son père était avocat), il a reçu une éducation religieuse de tradition catholique qu'il cherche également à transmettre à ses enfants et où la notion d'effort n'est pas absente : "bien sûr, mes parents accordaient de l'importance à la notion d'effort... "Il faut être dur au mal", par exemple. Mais cela ne s'est jamais appliqué au sport, plutôt aux efforts intellectuels, et notamment au travail scolaire mais toujours de façon très raisonnable». Son parcours scolaire et professionnel attestent si ce n'est d'un goût, au moins d'une pratique de l'ascèse intellectuelle et physique : "école privée (Jésuites) pendant 12 ans puis baccalauréat puis fac de médecine... J'ai reçu une éducation qui me paraissait trop sévère à l'époque... Malgré ce, je crois qu'ils ont réussi à me transmettre toutes les valeurs utiles, de rigueur, de travail, 
d'honnêteté ainsi que des valeurs religieuses (je suis catholique pratiquant)". La course à pied, puis l'ultrafond semblent offrir à Cyrille un terrain privilégié d'expression et de renforcement de ses dispositions à l'effort demandé par ces sports d'endurance.

La pratique de l'ultrafond permet tout à la fois l'expression de dispositions à l'effort extrême et leur renforcement dans la mesure où elle demande une préparation ascétique et rigoureuse ${ }^{16}$ qui contamine l'ensemble des sphères de la vie quotidienne: loisirs, alimentation, principes éducatifs, choix professionnels... Certains coureurs s'investissent également fortement dans un mode de sociabilité très sportif (groupe de coureurs du marathon des sables, club d'athlétisme spécialisé en ultrafond, etc.) renforçant leur identité de coureur d'ultrafond.

\section{Des ascètes improbables : travail de transformation de soi}

L'entrée dans l'ultrafond semble aussi pour certains coureurs être l'expression d'une rupture, plus ou moins prononcée, avec leurs habitudes alimentaires, leur rythme de vie, leurs pratiques culturelles. Il n'est d'ailleurs pas anodin de constater que l'expérience du marathon des sables s'effectue souvent pour ces coureurs au passage de la quarantaine. Dans un contexte de crise des identités (Dubar, 2000), cette course semble offrir à certains coureurs la possibilité de se "construire" (ou "reconstruire») une identité par un travail de transformation de soi (voire d'alternation - Berger \& Luckmann, 1989 ; Mennesson, 2004) recouvrant trois dimensions : physique, psychique et sociale (Darmon, 2003).

D'un point de vue physique, la transformation de soi est parfois très prononcée. La perte de poids engendrée par ou pour la pratique permet certes une amélioration des performances, mais modèle aussi le corps. Celui-ci participe directement à l'expression identitaire des coureurs (Detrez, 2002). L'importance du corps et des transformations engendrées par la pratique s'exprime d'ailleurs avec acuité dans les périodes de blessures (Allen-Collinson, 2003). C'est pourquoi il est important d'en prendre soin en surveillant son alimentation ${ }^{17}$, en étant

\footnotetext{
16 Selon les participants au marathon des sables, les trois éléments les plus importants pour améliorer son niveau de pratique sont : la régularité et la planification des entraînements ainsi qu'une bonne hygiène de vie.

$1760 \%$ des coureurs interrogés surveillent souvent voire très souvent leur alimentation et $37 \%$ suivent un régime spécifique en période de compétition.
} 
attentif aux petites douleurs musculaires ou articulaires et en procédant à une préparation minutieuse pour le marathon des sables (badigeonnage des pieds quelques semaines avant le départ afin de réduire le risque d'ampoules, collage de bandes d'élastoplaste aux endroits susceptibles de souffrir de frottements dus notamment au sac à dos...).

Exemple de Luc : "J'ai perdu 12 des $17 \mathrm{~kg}$ que j'ai pris quand j'ai arrêté de fumer! Le sport m'a aidé à me modérer et donné envie de faire attention. Après une course ou un bon entraînement, on n'a pas envie de boire d'alcool. Après un $100 \mathrm{~km}$ je mets 4 jours avant d'avoir envie d'un verre de vin, et à force on se dit que si l'organisme n'en veut pas, c'est que c'est pas bon et du coup on en prend de moins en moins... Ma femme m'a soutenu quand j'ai arrêté de fumer, disant que le poids n'était pas grave pour l'instant, le principal était d'arrêter, le poids on y remédierait plus tard! Plus tard tout allait bien, j'étais toujours aussi tonique, rien n'avait changé mis à part le volume! Mais un jour j'ai pensé que N. [son épouse] n'était pas forcément heureuse d'avoir une baleine dans son lit! Et tout ça, je savais qu'en plus de réussir des "exploits", d'être en bonne santé, de décrasser mes poumons (...), de prendre du plaisir, allait m'aider à dégonfler! J'étais pas obsédé par mon poids, mais maintenant j'en parle beaucoup parce que je croyais peut-être pas arriver à ce résultat et j'en suis d'autant plus fier. Je ne compte pas ressembler à un Apollon, mais $72 \mathrm{~kg}$ pour 1,73 m serait parfait. Je suis à 74,4».

Cette transformation physique participe à la définition des repères individuels en matière de santé et de bien-être (Vigarello, 1993). En privilégiant les qualités d'endurance, ces épreuves soulignent l'envie de durer et de vieillir en bonne santé. Le corps change (s'affine et se muscle) sous l'effet conjugué de l'entraînement et d'une hygiène de vie plus stricte. Cet aspect visible est d'autant plus important pour le coureur que le changement s'avère radical (perte parfois de plus de 20 $\mathrm{kg}$, arrêt de la cigarette, consommation réduite d'alcool...).

Cette transformation de soi comporte aussi une dimension mentale et morale plus ou moins fortement soulignée par les coureurs. Ils insistent quasi unanimement sur l'importance fondamentale du mental sur ce genre d'épreuve de très longue durée. Le mental prendrait le relais du physique pour ne pas lâcher et aller jusqu'au bout. Ainsi, les coureurs éprouvent leur capacité à aller au-delà de leurs souffrances et semblent transformer leur vision des obstacles qu'ils peuvent être amenés à surmonter dans leur vie quotidienne. Les longues heures de course à 
pied, dans le désert avec des conditions difficiles, semblent propices à la réflexion sur soi et à une véritable introspection. Plus les coureurs sont éloignés des enjeux chronométriques, plus ces transformations mentales prennent de l’importance. En véritables «pèlerins " (Barthélémy, 1999), ils explorent leurs capacités mentales à se dépasser dans une course en solitaire et en auto-suffisance. L'effort physique apparaît pour ces coureurs comme un des chemins de la connaissance de soi. Ils y attachent une quasi spiritualité.

Exemple de Michel : "Ma conception [de l'effort] a évolué en ce sens que j'ai pris conscience qu'avec suffisamment d'application, de préparation, de volonté et de patience, il était possible de franchir des étapes et d'arriver sur des distances propres à en décourager plus d'un... Le mental est primordial... La course à pied est pour moi un instant privilégié. Celui de pouvoir me dépasser et d'aller au bout d'un but qui me fait garder confiance en moi, celui d'éprouver des sensations rares de partage et d'observation de la nature, celui de voir plus loin vers de lointains objectifs et de vivre des moments d'émotion intense où la perception est aiguisée et très vive. Je cours très souvent en solitaire dans des endroits reculés et les moments sont propices à la réflexion et au souvenir. C'est pour moi un engagement et une forme de foi où ma mémoire reste vive... C'est le seul sport où je me retrouve véritablement avec moi-même sans rien devoir par ailleurs".

S'il est important de se prouver à soi-même cette capacité à se dépasser, le regard des autres joue un rôle primordial. Entre "vertus" et " folies", les efforts de ces marathoniens des sables attirent l'attention et participent à la définition même de leur identité sociale. L'image véhiculée à leur égard, soulignant leur courage et leur capacité à résister à la chaleur et à la souffrance, semble toujours positive. La reconnaissance sociale liée à la réussite d'une telle aventure est loin d'être négligeable comme en atteste, pour une part, sa forte médiatisation tant au niveau local que national ${ }^{18}$. L'idée qu'un tel défi est accessible au commun des mortels, sous conditions d'entraînement et de volonté, valorise d'autant plus ceux qui s'y astreignent.

\section{FRÉDÉRIC - né en 1965 - 163 ème au marathon des sables 2003 \\ Une véritable transformation physique et sociale}

\footnotetext{
18 Concernant l'édition 2004 du marathon des sables, les organisateurs ont dénombré un total de 234 articles de presse répartis de la façon suivante : 145 dans la presse régionale, 30 dans la presse nationale et 59 dans la presse internationale.
} 
Frédéric a commencé la course à pied en 1999 alors qu'il n'avait jamais fait de sport. Son envie de perdre du poids va être à l'initiative de ses premiers footings : "lorsqu'à 34 ans, on pèse $100 \mathrm{~kg}$, on a une fréquence cardiaque au repos de 85 pulses, une tension artérielle de 18 et un taux de cholestérol à 2.45, on se dit qu'il est temps de faire quelque chose avant d'avoir un sérieux problème de santé. J'ai donc pris "de bonnes résolutions"». Tout en perdant du poids, il accumule les kilomètres et les compétitions. En 2003, lors de son premier marathon des sables, il pèse $80 \mathrm{~kg}$ pour $1,83 \mathrm{~m}$ et a déjà pris part à des courses allant de $10 \mathrm{~km}$ à $100 \mathrm{~km}$. Il sera même à cette occasion membre de l'équipe "Jogging International ${ }^{19}$ » ce qui médiatisera ${ }^{20}$ plus sa participation.

Cet engouement pour l'ultrafond l'amène à toujours se lancer de nouveaux défis. Ainsi, après avoir réussi son premier marathon des sables, il prépara l'Ultra Trail du Mont-Blanc $(150 \mathrm{~km}$ de course avec $8000 \mathrm{~m}$ de dénivelé positif). Bien qu'il ait été conseillé dans ses entraînements par un entraîneur qualifié, il ne terminera pas cette course. Ce fut pour lui un échec et l'occasion de rebondir: "Tout abandon est vécu d'abord comme une grosse désillusion, une grande déception mais aussi comme une épreuve qui doit permettre de se remettre en cause et d'analyser les erreurs commises pour repartir encore plus fort. J'ai eu un coup au moral durant 15 jours puis j'ai décidé de me lancer un autre défi : courir un 24 heures. J'ai toujours en tête de refaire l'UTMB en août prochain avec la ferme ambition de faire le tour complet".

L'ultrafond a transformé son corps en particulier par la perte de poids réalisé : "depuis que je pratique un sport (depuis 1999), ma vie a changé. Je pèse $20 \mathrm{~kg}$ de moins. Ma tension artérielle est passée de 18/10 à 12/7. Je ne suis plus malade (un rhume tous les 2 ans environ). Mes analyses sanguines sont à encadrer». Mais bien plus, cette transformation prend aussi un caractère social dans la mesure où la course à pied rythme son temps de loisir, influence ses choix éducatifs ("j'attache beaucoup d’importance à ce que [mes enfants] fassent du sport et s'occupent de leur corps. Je considère que le corps humain est une machine formidable qui nécessite un peu d'attention. Commencer jeune, c'est garantir un capital santé pour le restant de ses jours. J'ai découvert un peu tard ce trésor mais je remercie tous les jours mon corps

\footnotetext{
19 Il s'agit d'une revue qui fait chaque année un appel à candidature afin d'être sponsorisé pour le marathon des sables en faisant partie de l'équipe du nom de la revue " Jogging International ".

20 Un article comptant les aventures des membres de cette équipe est paru dans le numéro 224, juin 2003, de Jogging International, pp.10-14.
} 
de me porter comme il le fait ") et pourrait être à l'origine de nouveaux choix professionnels ("j'étudie la possibilité d'ouvrir un magasin d'articles de sport spécialisé dans le running »).

L'entourage est souvent admiratif des performances réalisées. L'image qui est ainsi renvoyée au coureur par son milieu familial, professionnel et amical renforce l'importance d'un tel engagement. Cette identité de " sportif de l'extrême " capable de relever des défis exceptionnellement difficiles est incontestablement valorisante. Les participants au marathon des sables apparaissent " extraordinaires " aux yeux des gens " ordinaires". Courage, volonté, détermination et dépassement de soi ${ }^{21}$ participent pleinement à la définition de leur identité personnelle et professionnelle (Kay, Laberge, 2002).

\section{Conclusion}

Les modes d'entrée dans l'ultrafond se différencient essentiellement en fonction de l'histoire sportive des coureurs et de conditions sociales particulières favorisant un tel engagement. À la recherche de nouveauté, certains coureurs, aux dispositions sportives marquées par les sports d'endurance, trouveront dans l'ultrafond un parfait terrain d'expression de leurs qualités physiques et morales. Leur entrée dans l'ultrafond s'inscrit dans la continuité d'un parcours en course à pied relativement linéaire où les distances parcourues se sont progressivement allongées au fil des années. Ils font preuve d'un rapport très compétitif à l'ultrafond dans la mesure où, faisant partie des coureurs les mieux classés, ils cherchent constamment à améliorer leurs performances. D'autres coureurs, sportifs sur le tard et a priori moins disposés à s'engager sur ce type d'épreuve, semblent marquer, par leur entrée dans l'ultrafond, une véritable rupture avec leurs conditions sociales d'existence. Ils se lancent très rapidement sur de très longues distances mais ne s'intéressent que peu aux objectifs de classement.

Loin d'être anodin, un tel engagement marque les histoires de vie amenant des transformations physiques, mentales et/ou sociales. L'investissement temporel et énergétique que demande une telle 
activité ne peut, semble-t-il, rester sans conséquences dans la mesure où il contamine l'ensemble des sphères de l'existence. Une des questions essentielles restant en suspend est celle de connaître l'impact sur la longue durée d'un tel engagement. Les parcours biographiques sont-ils marqués durablement par cette expérience? Constitue-t-elle juste une période transitoire ou l'amorce d'une "nouvelle " vie ? Les incidences, à long terme, de tels engagements dépendent sans doute, dans des proportions variables, du degré de changement de dispositions initiales et de conditions d'existence plus ou moins adaptées aux efforts de très longue durée.

\section{RÉFÈRENCES}

ALLEN-COLLINSON (J.), 2003. "Running into injury time: distance running and temporality", Sociology of sport journal, 20, pp.331-350.

BADDELEY (M.) (dir), 2002. Sports extrêmes, sportifs de l'extrême. La quête des limites. Genève, Georg.

BARTHÉLÉMY (M.), 1998. "Le goût de l'extrême: passion et souffrance dans les aventures organisées ». In : BROMBERGER (C.) (Éd.), Passions ordinaires, Paris, Bayard, pp.477-495.

BARTHÉLÉMY (M.), 1999. "Les expériences multiples de l'endurance ", Sociétés, 64, pp.43-49.

BARTHÉLÉMY (M.), 2002. "Approche ethnographique d'une aventure extrême : le marathon des sables ", Sport med, 141, pp.6-8.

BERGER (P.), LUCKMANN (T.), 1989. La construction sociale de la réalité, Paris, Méridiens Klincksieck.

BERTAUX (D.), 1997. Les récits de vie, Paris, Nathan.

BOURDIEU (P.), 1980. Le sens pratique, Paris, Éditions de Minuit.

DARMON (M.), 2003. Devenir anorexique, Paris, La découverte.

DETREZ (C.), 2002. La construction sociale du corps, Paris, Seuil.

DUBAR (C.), 2000. La crise des identités, Paris, PUF.

EHRENBERG (A.), 1991. Le culte de la performance, Paris, Calman Lévy.

KAY J., LABERGE S. (2002). The 'new' corporate habitus in adventure racing. International Review for the Sociology of Sport, 37/1, 17-36.

LAHIRE (B.), 1998. L'homme pluriel. Paris, Nathan.

LAHIRE (B.), 2002. Portraits sociologiques, Paris, Nathan.

LE BRETON (D.), 1991. Passion du risque, Paris, Métailié. 
MENNESSON (C.), 2004. " Être une femme dans un sport "masculin" ", Sociétés Contemporaines, 55, pp.69-90.

MIGNON (P.), TRUCHOT (G.), (dir) 2002. Les pratiques sportives en France, Paris, Ministère des Sports et INSEP.

POCIELLO (C.), 1981. " La force, l'énergie, la grâce et les réflexes. Le jeu complexe des dispositions culturelles et sportives ». In : POCIELLO (C.) (Éd.), Sports et société.,Paris, Vigot, pp.171-237.

QUÉVAL (I.), 2004. S’accomplir ou se dépasser. Paris, Gallimard.

SEGALEN (M.), 1994. Les enfants d'Achille et de Nike. Une ethnologie de la course à pied ordinaire. Paris, Métailié.

SMITH (S.L.), 2000. "British nonelite road running and masculinity. A case of 'running repairs'?", Men and masculinities, 3/2, pp.187-208.

VIGARELLO (G.), 1993. Le sain et le malsain. Santé et mieux-être depuis le Moyen-Âge. Paris, Seuil. 


\section{Annexe 1 - Enquête par questionnaire}

Réalisée le 8 avril 2004. 123 des 186 concurrents français ont répondu (taux de réponse de $66 \%$ ).

Quelques données descriptives de la population interrogée :

\begin{tabular}{|c|c||c|c||c|c|c|c|}
\hline \multicolumn{2}{|c||}{ Sexe } & \multicolumn{5}{c|}{$\begin{array}{c}\text { Situation } \\
\text { familiale }\end{array}$} & \multicolumn{4}{c|}{ Profession } & $\%$ \\
\hline & $\%$ & & $\%$ & & $\%$ & & \\
& & & & & & \\
homme & $\mathbf{9 0 , 2 4}$ & seul(e) & 19,51 & $\begin{array}{l}\text { artisans, commerçants } \\
\text { et chefs d'entreprise }\end{array}$ & 4,07 & ouvriers & 8,94 \\
\hline femme & 9,76 & en couple & $\mathbf{7 8 , 8 6}$ & cadres & $\mathbf{2 1 , 1 4}$ & retraités & 1,63 \\
\hline Total & 100,00 & $\begin{array}{l}\text { Eép. } \\
\text { manquante* }\end{array}$ & 1,63 & prof. intermédiaires & $\mathbf{3 3 , 3 3}$ & sans profession & 2,44 \\
\hline
\end{tabular}

\begin{tabular}{|l|c|c|c|c|}
\hline & Moyenne & Écart-type & Minimum & Maximum \\
\hline Années de pratique en course à pied & 14,28 & 7,41 & 2 & 44 \\
\hline $\begin{array}{l}\text { Nombre de participations au } \\
\text { marathon des sables }\end{array}$ & 1,87 & 2,03 & 1 & 16 \\
\hline Âge & 43,44 & 7,95 & 28 & 66 \\
\hline
\end{tabular}




\section{Annexe 2 - Enquête par interview}

Réalisée de mai 2003 à juillet 2005. 50 participants au marathon des sables 2003, 2004 et/ou 2005 ont été interviewés. L'ensemble des enquêtés se répartit de la manière suivante :

\begin{tabular}{|l|c|c|c|}
\hline Classement au marathon des sables / Sexe & Homme & Femme & Total \\
\hline $\mathbf{1}^{\text {er }}$ quart du classement & 16 & 3 & $\mathbf{1 9}$ \\
\hline $\mathbf{2}^{\text {ème }}$ quart du classement & 10 & 1 & $\mathbf{1 1}$ \\
\hline $3^{\text {ème }}$ quart du classement & 8 & 1 & $\mathbf{9}$ \\
\hline dernier quart du classement & 6 & 5 & $\mathbf{1 1}$ \\
\hline Total & $\mathbf{4 0}$ & $\mathbf{1 0}$ & $\mathbf{5 0}$ \\
\hline
\end{tabular}

Quelques données descriptives de la population interviewée :

\begin{tabular}{|c|c|c|c|}
\hline & Homme & Femme & Total \\
\hline \multicolumn{4}{|c|}{$\hat{\mathbf{A} G E}$} \\
\hline-40 ans & 15 & 3 & 18 \\
\hline 40 à 50 ans & 16 & 4 & 20 \\
\hline+50 ans & 9 & 3 & 12 \\
\hline Total & 40 & 10 & 50 \\
\hline \multicolumn{4}{|c|}{ NOMBRE D'ANNÉES DE PRATIQUE DE LA COURSE À PIED } \\
\hline-10 ans & 12 & 3 & 15 \\
\hline 10 à 15 ans & 11 & 4 & 15 \\
\hline+15 ans & 17 & 3 & 20 \\
\hline Total & 40 & 10 & 50 \\
\hline
\end{tabular}

